\title{
BMJ Open Helmet Non-Invasive Ventilation for COVID-19 Patients (Helmet-COVID): study protocol for a multicentre randomised controlled trial
}

\begin{abstract}
Yaseen M Arabi (D) , ${ }^{1,2}$ Haytham Tlayjeh, ${ }^{1,2}$ Sara Aldekhyl, ${ }^{1,2}$ Hasan M Al-Dorzi, ${ }^{1,2}$ Sheryl Ann Abdukahil, ${ }^{1,2}$ Mohammad Khulaif Al Harbi, ${ }^{2,3}$ Husain Al Haji, ${ }^{4}$ Mohammed Al Mutairi, ${ }^{4}$ Omar Al Zumai, ${ }^{4,5}$ Eman Al Qasim, ${ }^{1,2}$ Wedyan AI Wehaibi, ${ }^{1,2}$ Saad Al Qahtani, ${ }^{1,2}$ Fahad Al-Hameed, ${ }^{6,7}$ Jamal Chalabi, ${ }^{8,9}$ Mohammed Alshahrani, ${ }^{10}$ Talal Albrahim, ${ }^{11}$ Abdulrahman Alharthy, ${ }^{12}$ Ahmed Mady, ${ }^{12,13}$ Abdulhadi Bin Eshaq,${ }^{14}$ Ali A Al bshabshe (D) , ${ }^{15,16}$ Zohair Al Aseri, ${ }^{17,18}$ Zainab Al Duhailib, ${ }^{19}$ Ayman Kharaba, ${ }^{20}$ Rakan Alqahtani, ${ }^{21}$ Adnan Al Ghamdi, ${ }^{22}$ Ali Altalag, ${ }^{22}$ Khalid Alghamdi, ${ }^{23}$ Mohammed Almaani, ${ }^{24}$ Haifa Algethamy, ${ }^{25}$ Ahmad Al Aqeily, ${ }^{4}$ Faisal Al Baseet, ${ }^{4}$ Hashem Al Samannoudi, ${ }^{4}$ Mohammed Al Obaidi, ${ }^{4}$ Yassin T Ismaiel, ${ }^{4}$ Abdulrahman A Al-Fares ${ }^{26}$
\end{abstract}

To cite: Arabi YM, Tlayjeh $\mathrm{H}$, Aldekhyl S, et al. Helmet Non-Invasive Ventilation for COVID-19 Patients (HelmetCOVID): study protocol for a multicentre randomised controlled trial. BMJ Open 2021;11:e052169. doi:10.1136/ bmjopen-2021-052169

- Prepublication history and additional supplemental material for this paper are available online. To view these files, please visit the journal online. (http://dx.doi.org/10.1136/ bmjopen-2021-052169).

Received 07 April 2021 Accepted 29 July 2021
Check for updates

(C) Author(s) (or their employer(s)) 2021. Re-use permitted under CC BY-NC. No commercial re-use. See rights and permissions. Published by BMJ.

For numbered affiliations see end of article.

Correspondence to Dr Yaseen M Arabi; yaseenarabi@yahoo.com

\section{ABSTRACT}

Introduction Non-invasive ventilation (NIV) delivered by helmet has been used for respiratory support of patients with acute hypoxaemic respiratory failure due to COVID-19 pneumonia. The aim of this study was to compare helmet NIV with usual care versus usual care alone to reduce mortality.

Methods and analysis This is a multicentre, pragmatic, parallel randomised controlled trial that compares helmet NIV with usual care to usual care alone in a 1:1 ratio.

A total of 320 patients will be enrolled in this study.

The primary outcome is 28-day all-cause mortality. The primary outcome will be compared between the two study groups in the intention-to-treat and per-protocol cohorts. An interim analysis will be conducted for both safety and effectiveness.

Ethics and dissemination Approvals are obtained from the institutional review boards of each participating institution. Our findings will be published in peer-reviewed journals and presented at relevant conferences and meetings.

Trial registration number NCT04477668.

\section{INTRODUCTION}

The novel SARS-CoV-2 has led to a pandemic resulting in over 181 million cases and approximately 4.4 million fatalities as of 17 August 2021. ${ }^{1}$ The resulting COVID-19 leads to severe pneumonia and acute respiratory distress syndrome (ARDS) among other organ injuries. ${ }^{2}$ ARDS may occur in up to $5 \%$ of infected patients. ${ }^{3-5}$ Early in the pandemic, invasive mechanical ventilation was widely used because of concerns about non-invasive ventilation (NIV) safety and efficacy. However,

\section{Strengths and limitations of this study}

This trial compares helmet non-invasive ventilation to usual care for respiratory support of patients with acute hypoxaemic respiratory failure due to COVID-19 pneumonia.

- The trial is a multicentre, pragmatic, parallel randomised controlled trial.

- The main limitation is the unblinded design due to the nature of the intervention.

NIV use increased with time, including mask NIV and helmet NIV.

NIV has been shown to have physiological benefits in patients with acute hypoxaemic respiratory failure (AHRF) secondary to pulmonary oedema, atelectasis or pneumonia. ${ }^{6}$ It has been shown to improve arterial oxygenation by increasing functional residual capacity, shifting the tidal volume to a more compliant part of the pressure-volume curve, thus reducing both the work of breathing and the risk of tidal opening and closure of the airways. ${ }^{7}$ NIV is commonly provided through nasal or oronasal interfaces. The resulting aerosol generation may increase the risk of transmission of pathogens to healthcare providers, raising concerns about the use of NIV in patients with viral pneumonia. ${ }^{8}$ Helmet NIV has been used for AHRF including patients with COVID-19 pneumonia. The helmet surrounds the patient's entire head to provide positive pressure and 
supply oxygen and is sealed with a soft, airtight collar that wraps around the neck. Due to this design, it has advantages over the nasal and oronasal interfaces. These include less air leaks, no skin or nasal bridge skin injuries, no eye irritation, fitting for different facial contours ${ }^{9}$ and hypothetically less dissemination of aerosols in the environment. However, helmet interface may be associated with increase in dead space (especially if the settings are not used appropriately), claustrophobia, discomfort, and difficulty in access for suction and feeding.

Evaluation of helmet NIV as a respiratory support modality started more than two decades ago. ${ }^{10}$ Helmet NIV has been investigated as a treatment for different forms of AHRF in adults in various settings, such as prehospital ambulance, emergency department and intensive care unit (ICU). ${ }^{711-15}$ However, earlier clinical studies are relatively scarce and mostly small in size, and often use improvement in oxygenation and intubation rate as primary outcomes. ${ }^{6}$

However, evidence on helmet NIV for AHRF is growing. A systematic review of randomised controlled trials (RCTs) and observational studies published before June 2016 found 11 studies involving 621 patients. ${ }^{16}$ Compared with controls, the use of a helmet was associated with lower hospital mortality, intubation rate and complications. ${ }^{16}$ There was no significant difference in gas exchange and ICU stay. ${ }^{16}$ A meta-analysis of four RCTs (377 patients) showed that helmet NIV significantly increased the ratio of arterial oxygen partial pressure to fraction of inspired oxygen $\left(\mathrm{PaO}_{2}: \mathrm{FiO}_{2}\right)$ and decreased arterial carbon dioxide levels, intubation rate and in-hospital mortality compared with standard oxygen therapy. ${ }^{17}$ In a more recent systematic review and network meta-analysis that included 25 studies (published up to April 2020) with 3804 patients with AHRF, mortality and intubation rate were lower with helmet NIV compared with standard oxygen by more than $50 \%$, while the effects of mask NIV and high-flow nasal oxygen were modest compared with those of standard oxygen. ${ }^{18}$ Helmet NIV was superior to both mask NIV and high-flow nasal oxygen, while mask NIV and high-flow nasal oxygen were not different in their effects on mortality and intubation rate. ${ }^{18}$ One study reported the cost-effectiveness of helmet NIV compared with mask NIV. ${ }^{19}$

Data on helmet NIV in AHRF related to COVID-19 are emerging. A recent RCT conducted in four Italian ICUs on patients with COVID-19 and moderate-to-severe AHRF found that treatment with helmet NIV did not result in significantly fewer days of respiratory support at 28 days from randomisation (primary outcome) as compared with high-flow nasal oxygen alone (mean difference, 2 days; $95 \%$ CI -2 to 6 ; $\mathrm{p}=0.26) .{ }^{20}$ Nevertheless, the intubation rate was significantly lower in the helmet NIV group compared with the high-flow nasal oxygen group $(30 \%$ vs $51 \%, \mathrm{p}=0.03) .{ }^{20}$ Additionally, the median number of days free of invasive mechanical ventilation within 28 days was significantly higher in the helmet NIV group than in the high-flow nasal oxygen group (28 vs 25 days; mean difference, 3 days; $95 \%$ CI 0 to $7 ; \mathrm{p}=0.04) .{ }^{20}$ The hospital mortality was $24 \%$ in the helmet NIV group and $25 \%$ in the high-flow nasal oxygen group. ${ }^{20}$

As the efficacy of helmet NIV to improve outcomes in severe AHRF due to COVID-19 pneumonia has not been clearly established, the aim of this study was to compare helmet NIV with usual care versus usual care alone to reduce 28-day all-cause mortality. We hypothesise that helmet NIV will reduce 28-day all-cause mortality in patients with suspected or confirmed severe COVID-19 pneumonia and AHRF.

\section{METHODS AND ANALYSIS \\ Trial design}

This is an investigator-initiated, pragmatic parallel RCT that will compare helmet NIV with usual care to usual care alone in 1:1 ratio in patients with suspected or confirmed COVID-19 pneumonia and AHRF. Randomisation is performed using a computer-generated schedule using variable block sizes ( 4 or 6 ) and is stratified by site. The trial is sponsored by King Abdullah International Medical Research Center, Riyadh, Saudi Arabia, has been registered with ClinicalTrials.gov and is conducted across multiple centres in Saudi Arabia and Kuwait. Training and in-service education on helmet NIV use as well as on protocol implementation are provided to all participating centres. The competency of the bedside respiratory therapists is supervised by experienced respiratory care supervisors and intensivists.

\section{Sample size}

In a large observation study of patients with AHRF and ARDS, hospital mortality was $34.9 \%$ (95\% CI $31.4 \%$ to $38.5 \%$ ) for patients with mild ARDS, $40.3 \%$ (95\% CI $37.4 \%$ to $43.3 \%$ ) for those with moderate ARDS and $46.1 \%$ (95\% CI $41.9 \%$ to $50.4 \%$ ) for those with severe ARDS. ${ }^{21}$ A systematic review found an overall pooled mortality estimate among 10815 patients with ARDS due to COVID-19 to be $39 \%$ (95\% CI $23 \%$ to $56 \%) .{ }^{22}$ Considering a mortality rate of $40 \%$ in patients with COVID-19 pneumonia and moderate to severe ARDS treated with usual care, we calculated that enrolment of 304 patients (152 in each group) would provide the study with $80 \%$ power to demonstrate an absolute difference of $15 \%$ in the primary outcome between the usual care group and the helmet NIV group at a two-sided alpha level of 0.05 . To account for $5 \%$ loss to follow-up, the total number of patients required for the trial is 320 patients.

\section{Participant eligibility}

The trial will enrol ICU patients with suspected ${ }^{23}$ or confirmed COVID-19 pneumonia who have AHRF. Detailed inclusion and exclusion criteria can be found in table 1 . 
Table 1 Eligibility criteria

Inclusion criteria 1 . Suspected or confirmed COVID-19.*

2. Aged $\geq 14$ years old. ICUs that use other age cut-off for adult patients will adhere to their local standard (16 or 18 years).

3. Acute hypoxaemic respiratory failure based on $\mathrm{PaO}_{2}: \mathrm{FiO}_{2}$ ratio $<200$ despite supplemental oxygen with a partial or non-rebreathing mask at a flow rate $>10 \mathrm{~L} / \mathrm{min}$ or higher.

4. Intact airway protective gag reflex.

5. Able to follow instructions.

Exclusion criteria $\begin{aligned} & \text { 1. Prior intubation during this hospital } \\ & \text { admission. }\end{aligned}$
2. Cardiopulmonary arrest.
3. Glasgow Coma Scale score of $<12$.
4. Tracheostomy.
5. Upper airway obstruction.
6. Active epistaxis.
7. Requirement for more than one
vasopressor to maintain mean arterial
pressure $>65$ mm Hg.
8. Pregnancy.
9. Imminent intubation.
10. Patients with do-not-intubate orders
(or equivalent).
11. Enrolled in another trial for which
coenrolment is not approved,
including trials on mechanical
ventilation.
12. Patients already treated with helmet.
13. Patients with chronic carbon dioxide
retention (PaCO $>45)$.
14. Previous enrolment in this trial.
15. The primary cause of respiratory
failure is not heart failure as judged
by the treating team.

${ }^{*}$ A suspected/probable COVID-19 case is defined as at least two of the following symptoms: fever (measured or subjective), chills, rigours, myalgia, headache, sore throat, new olfactory and taste disorder(s) or at least one of the following symptoms: cough, shortness of breath or difficulty breathing, or severe respiratory illness with at least one of the following: clinical or radiographical evidence of pneumonia or ARDS and no alternative more likely diagnosis. A confirmed COVID-19 case is defined as detection of SARS-CoV-2 RNA in a respiratory specimen using a molecular amplification detection test such as RT-PCR (https://wwwn.cdc. gov/nndss/conditions/coronavirus-disease-2019-covid-19/casedefinition/2020/).

$\mathrm{ICU}$, intensive care unit; $\mathrm{PaO}_{2}: \mathrm{FiO}_{2}$, ratio of arterial oxygen partial pressure to fraction of inspired oxygen; RT-PCR, reverse transcription PCR.

\section{Informed consent}

Informed consent will be obtained from the potential trial participants or their surrogate decision makers. A hybrid model of consent will be used where a priori consent is obtained, if possible; otherwise, delayed consent model will be obtained as per local approvals. The first patient was enrolled in February 2021. As of 29 June 2021, a total of 199 patients were enrolled from five sites. There are several sites that are processing institutional review board (IRB) and regulatory approvals.

\section{Patient and public involvement}

There was no patient or public involvement in the conception, design or conduct of the study, or the writing or editing of this paper. However, patient comfort and experience as well as compliance to the intervention were taken into consideration and data on these were collected.

\section{Trial interventions}

Helmet group

A helmet (Subsalve, USA, or its equivalent) which is made of transparent latex-free polyvinyl chloride will be applied to patients randomised to the intervention group as per the study protocol, which considers the manufacturer instructions. It will be connected to an ICU ventilator in pressure support (PS) mode with positive end-expiratory pressure (PEEP) using a conventional respiratory circuit joining two port sites to allow inspiratory and expiratory flows. The starting settings is PS of $8-10 \mathrm{~cm} \mathrm{H}_{2} \mathrm{O}$, PEEP of $10 \mathrm{~cm} \mathrm{H}_{2} \mathrm{O}$ with $\mathrm{FiO}_{2}$ of $100 \%$, targeting flow rate of $\geq 50 \mathrm{~L} / \mathrm{min}$ with an inspiratory rise time of $50 \mathrm{~ms}$ and end flow/cycling off of $50 \%$ of maximal inspiratory flow. PEEP may be increased by $2 \mathrm{~cm}$ every $3 \mathrm{~min}$ to achieve oxygen saturation $\left(\mathrm{SpO}_{2}\right) \geq 90 \%$ on $\mathrm{FiO}_{2} \leq 60 \%$, and PS can be increased by $2 \mathrm{~cm}$ every $3 \mathrm{~min}$ to achieve respiratory rate of $\leq 25$ breaths/min and disappearance of accessory muscle activity. The maximal allowed PS+PEEP is $30 \mathrm{~cm} \mathrm{H}_{2} \mathrm{O}$. Interruptions of helmet should be avoided or kept at minimum at least in the first 48 hours. ${ }^{20}$ More details of helmet NIV application, set-up and weaning can be found in online supplemental file 1. Some patients may not tolerate helmet NIV. In that case, the physician or the respiratory therapist explains the procedure to the patient. Dexmedetomidine infusion may be used to improve comfort with the helmet NIV. Other intravenous sedatives such as benzodiazepines or intravenous narcotics should generally not be used. If the patient continues to be intolerant to the helmet, the patient can be managed according to the usual care. Detailed data about helmet NIV tolerance are collected.

\section{Control group}

In the control group, patients receive usual care according to the clinical practices of each site. This may include oxygen provided using standard oxygen devices, high-flow nasal oxygen or NIV provided by nasal mask, face mask or total mask.

\section{Endotracheal intubation}

The decision to intubate will be at the discretion of the treating team with no involvement from the research 
team. However, the protocol provides guidance on assessing patients for the need of endotracheal intubation throughout the study period (for both study groups: helmet NIV or usual care) according to the following general principles.

Enrolled patients should be assessed within 4 hours of enrolment (or sooner as required) and at frequent intervals for the following criteria, although the decision is usually not based on a single variable:

- Neurological deterioration (not attributed to sedation).

- Persistent or worsening respiratory failure of NIV (manifesting as $\mathrm{SpO}_{2}<88 \%$, respiratory rate $>36$ breaths $/ \mathrm{min}, \mathrm{PaO}_{2}: \mathrm{FiO}_{2}$ ratio $<100$ or persistent requirement of $\mathrm{FiO}_{2} \geq 70 \%$ ).

- Intolerance of face mask or helmet.

- Airway bleeding.

- Copious respiratory secretions.

- Respiratory acidosis with $\mathrm{pH}<7.25$

- Haemodynamic instability.

- Significant radiological worsening.

\section{Cointerventions}

Patients who require endotracheal intubation are managed by the primary team with lung protective strategy with tidal volumes of $6 \mathrm{~mL} / \mathrm{kg}$ of predicted body weight and titration of PEEP to achieve $\mathrm{SpO}_{2}$ of $88 \%-95 \%$ at the lowest possible $\mathrm{FiO}_{2}$. Daily interruption of sedation, awakening and breathing trials, and early mobilisation are performed as per the ICU standards. ${ }^{24}$ Management of COVID-19 is provided as per local protocols; physicians are advised to follow the clinical practice guidelines set by the Saudi Critical Care Society, ${ }^{25}$ the Surviving Sepsis Campaign $^{26} 27$ and the WHO. ${ }^{28}$ The study protocol does not mandate particular therapies; however, corticosteroids, immune modulators and antiviral therapy are all recorded. Conservative fluid management is recommended where neutral balance should be targeted and intravenous resuscitation should be reserved for shock treatment in both groups and fluid balance is recorded.

\section{Blinding}

Due to the nature of the study intervention, blinding is not be possible.

\section{Recruitment schedule and enrolment procedures}

Schedule of assessments is detailed in table 2. All non-intubated subjects with suspected or confirmed COVID-19 are screened on admission to the ICU. A screening log will be kept to monitor and report the size of the patient population from which eligible patients have been randomised. Coenrolment in other RCTs is permissible as long as inclusion in the other RCT would not confound the results of this trial and after discussion with the steering committees of the other studies.

\section{Data collection}

Baseline data on demographics, admission diagnosis and clinical information are collected. Clinical information include Acute Physiology and Chronic Health Evaluation (APACHE) II score, ${ }^{29}$ source of admission, ICU admission category (elective, emergency or non-surgical), ICU admission diagnosis and comorbidities (as defined by the APACHE II severity of illness scoring system). Daily data will be recorded until discharge from the ICU or 28 days after randomisation. We will collect data on the use of helmet including the tolerance of helmet (>1-hour use).

\section{Outcomes}

The primary outcome is 28-day all-cause mortality. Secondary outcomes are intubation rate within 28 days, ICU mortality, hospital mortality (censored at day 180), ICU-free days at day 28, invasive ventilation-free days at day 28, renal replacement therapy-free days at day 28 and vasopressor-free days at day 28. Safety outcomes include skin pressure injuries, barotrauma and serious adverse

\section{Table 2 Schedule of assessments in the trial}

\begin{tabular}{|c|c|c|c|c|c|}
\hline Task & Screening & Randomisation & Baseline & Days 1-28 & $\begin{array}{l}\text { 180-day } \\
\text { follow-up }\end{array}$ \\
\hline Assess eligibility to enter study & $x$ & & & & \\
\hline Assess ability to gain consent and follow-up & $\mathrm{x}$ & & & & \\
\hline Consent & $x$ & & & & \\
\hline Demographics and eligibility checklist & $\mathrm{x}$ & $\mathrm{x}$ & $\mathrm{x}$ & & \\
\hline Laboratory data & & & $x$ & $\mathrm{x}$ & \\
\hline Vital signs & & & $x$ & $\mathrm{x}$ & \\
\hline Vital status up to day 28 in the ICU & & & & $x$ & $\mathrm{x}$ \\
\hline Vital and functional status & & & & & $\mathrm{x}$ \\
\hline Discharge date from ICU, from hospital & & & & $x$ & $\mathrm{x}$ \\
\hline Adverse events & & & & $\mathrm{x}$ & \\
\hline Protocol violations & & & & $\mathrm{X}$ & \\
\hline
\end{tabular}

ICU, intensive care unit. 
events (including cardiovascular events and device complications).

Additionally, there will be a follow-up of enrolled patients at day 180 about vital status, functional status (EuroQoL-5D-5L) which is planned to be reported separately. For patients who have been discharged from hospital before day 180, follow-up will be conducted by telephone.

\section{Data analysis}

A formal statistical analysis plan will be agreed on and placed in the public domain before the study database is locked for the analysis of the primary outcome. The primary outcome will be compared in the intention-totreat and per-protocol cohorts (effectiveness analysis) using the $\chi^{2}$ test. Results will be reported as relative risk with $95 \%$ CI. Kaplan-Meier curves will be plotted to assess the time from enrolment to death and will be compared by means of the log-rank test. A two-tailed $p$ value of $<0.05$ will be considered to indicate statistical significance. SAS software V.9.2 will be used for all the analyses.

A priori analysis will be done for the following subgroups:

- Patients with moderate ARDS $\left(\mathrm{PaO}_{2}: \mathrm{FiO}_{2}\right.$ ratio 100-200) and patients with severe ARDS $\left(\mathrm{PaO}_{2}: \mathrm{FiO}_{2}\right.$ ratio $<100$ ).

- Obese patients (body mass index $>30 \mathrm{~kg} / \mathrm{m}^{2}$ ) and patients with body mass index of $\leq 30$.

- Patients aged $>65$ and $\leq 65$ years.

- APACHE II score higher or lower than the median of enrolled patients.

For the occasional randomised patient who is withdrawn from the trial and allows use of data, the patient's data will be included in the group to which he/she was allocated as per the intention-to-treat principle, and the reason of withdrawal will be documented.

\section{Trial management and monitoring}

The study steering committee members will be responsible for overseeing the conduct of the trial, for upholding or modifying study procedures as needed, addressing challenges with protocol implementation, formulating the analysis plan, reviewing and interpreting the data, and preparing the manuscript. This will be achieved through meetings (in-person or by conference calls) at least quarterly.

Several measures are taken to minimise, observe and document any potential safety concerns. First, any unexpected safety concerns will be reported immediately to the steering committee and IRB. Second, an independent data safety monitoring board will be monitoring the safety of the trial. Lastly, interim analyses will be conducted after recruiting one-third and two-thirds of the total patients, and the interim test statistics will be the primary outcome analysis for both safety and effectiveness. The data safety monitoring board will use formal stopping rules based on the primary endpoint of 28-day mortality. The trial may be stopped for safety $(p<0.01)$ or effectiveness $(p<0.001)$. There will be no plans to terminate the trial for futility. We will account for alpha spending by the O'Brien-Fleming method, and the final $\mathrm{p}$ value will be considered at 0.048 . The principles used in the conduct of safety monitoring and reporting in this trial are those outlined by Cook $e t$ $a l^{30}$

In this trial, reporting of serious adverse events will be restricted to events that are not captured as study outcome and are considered to be related to the helmet NIV (possibly, probably or definitely). ${ }^{30}$ These may include cardiovascular events (ie, cardiac arrest and hypotension with drop in blood pressure to systolic $<90 \mathrm{~mm} \mathrm{Hg}$ ) and device complications (ie, helmet deflation).

\section{ETHICS AND DISSEMINATION}

The study will be conducted according to the principles of the latest version of Good Clinical Practice and in accordance with all relevant local ethical, regulatory and legal requirements. A manuscript with the results of the primary study will be published in a peer-reviewed journal. Separate manuscripts will be written on secondary aims,

Table 3 List of ongoing registered RCTs on helmet NIV

\begin{tabular}{|c|c|c|c|c|c|}
\hline Trial & Registration & Interventions & Design & Countries & $\mathbf{N}$ \\
\hline Helmet-COVID & NCT04477668 & $\begin{array}{l}\text { Helmet versus usual } \\
\text { care }\end{array}$ & Multicentre RCT & $\begin{array}{l}\text { Saudi Arabia } \\
\text { and Kuwait }\end{array}$ & 320 \\
\hline $\begin{array}{l}\text { Comparison of High-Flow Nasal Oxygen, } \\
\text { Face-Mask NIV and Helmet NIV in } \\
\text { COVID-19 ARDS Patients }\end{array}$ & NCT04715243 & $\begin{array}{l}\text { High-flow nasal oxygen } \\
\text { versus helmet NIV } \\
\text { versus mask NIV }\end{array}$ & Multicentre RCT & Oman & 360 \\
\hline $\begin{array}{l}\text { Helmet CPAP vs High-Flow Nasal } \\
\text { Oxygen in COVID-19 }\end{array}$ & NCT04395807 & $\begin{array}{l}\text { High-flow nasal oxygen } \\
\text { versus helmet CPAP }\end{array}$ & Single-centre RCT & Sweden & 120 \\
\hline $\begin{array}{l}\text { High-Flow Nasal Oxygen vs CPAP } \\
\text { Helmet in COVID- } 19 \text { Pneumonia }\end{array}$ & NCT04381923 & $\begin{array}{l}\text { High-flow nasal oxygen } \\
\text { versus helmet CPAP }\end{array}$ & Single-centre RCT & USA & 200 \\
\hline $\begin{array}{l}\text { Early CPAP in COVID-19 Patients with } \\
\text { Respiratory failure (EC-COVID-RCT) }\end{array}$ & NCT04326075 & $\begin{array}{l}\text { Early helmet CPAP } \\
\text { versus usual care }\end{array}$ & Single-centre RCT & Italy & 900 \\
\hline
\end{tabular}

Helmet-COVID, Helmet Non-Invasive Ventilation for COVID-19; NIV, non-invasive ventilation; RCT, randomised controlled trial. 
and these will also be submitted for publication in peerreviewed journals as well.

\section{DISCUSSION}

The importance of this study stems from the current pandemic situation as different treatment modalities are being sought to answer important clinical questions. Available literature on the evaluation of helmet NIV as a respiratory support modality in patients with COVID-19 is limited. Table 3 provides a list of ongoing RCTs on helmet NIV. This study aimed to contribute to the existing literature and in turn influence clinical practice.

We planned our pragmatic trial to address whether using helmet NIV as the primary non-invasive respiratory support in patients with severe COVID-19, in addition to the commonly used high-flow nasal oxygen and mask NIV, improves outcome. By nature of this question, there is heterogeneity of the control group, as patients in this group could receive standard oxygen, high-flow nasal oxygen or mask NIV at the decision of the treating team. This approach is supported by a recent network meta-analysis of RCTs that showed only a modest effect of high-flow nasal oxygen and mask NIV on mortality or intubation rate compared with standard oxygen, while patients treated with helmet NIV had more than 50\% reduction in mortality and intubation rate compared with the other three modalities. ${ }^{18}$ In addition, this approach is likely to be more representative of usual practice in which patients may get oxygen therapy, high-flow nasal oxygen and NIV at different times during their acute illness. Given the fact that the use of helmet NIV has not been widespread across ICUs, we thought that the broader question addressed by our study might be more relevant to deciding whether to introduce this modality or not in a given ICU.

The main limitation to our study is inability to blind the given allocation due to the nature of the intervention.

\footnotetext{
Author affiliations

${ }^{1}$ Intensive Care Department, Ministry of National Guard Health Affairs, Riyadh, Saudi Arabia

${ }^{2}$ College of Medicine, King Saud bin Abdulaziz University for Health Sciences, King Abdullah International Medical Research Center, Riyadh, Saudi Arabia

${ }^{3}$ Department of Anesthesia, Ministry of National Guard Health Affairs, Riyadh, Saudi Arabia

${ }^{4}$ Respiratory Services Department, Ministry of National Guard Health Affairs, Riyadh, Saudi Arabia

${ }^{5}$ College of Applied Medical Sciences, King Saud bin Abdulaziz University for Health Sciences, Riyadh, Saudi Arabia

${ }^{6}$ Intensive Care Department, Ministry of National Guard Health Affairs, Jeddah, Saudi Arabia

${ }^{7}$ King Saud bin Abdulaziz University for Health Sciences, King Abdullah International Medical Research Center, Jeddah, Saudi Arabia

${ }^{8}$ Intensive Care Department, Ministry of National Guard Health Affairs, Al Ahsa,

Saudi Arabia

${ }^{9}$ King Saud bin Abdulaziz University for Health Sciences, King Abdullah International Medical Research Center, Al Ahsa, Saudi Arabia

${ }^{10}$ Departments of Emergency and Critical Care, Imam Abdulrahman Bin Faisal University, King Fahd Hospital of the University, Al Khobar, Saudi Arabia

${ }^{11}$ Department of Critical Care, Imam Abdulrahman Bin Faisal University, King Fahad Hospital of the University, Al Khobar, Saudi Arabia
}

${ }^{12}$ Intensive Care Department, King Saud Medical City, Riyadh, Saudi Arabia

${ }^{13}$ College of Medicine, Tanta University, Tanta, Egypt

${ }^{14}$ Intensive Care Department, King Khalid Hospital Najran, Najran, Saudi Arabia

${ }^{15}$ College of Medicine, King Khalid University, Abha, Saudi Arabia

${ }^{16}$ Intensive Care Department, Aseer Central Hospital, Abha, Saudi Arabia

${ }^{17}$ Departments of Emergency Medicine and Critical Care, College of Medicine, King

Saud University, Riyadh, Saudi Arabia

${ }^{18}$ College of Medicine, King Saud University, Riyadh, Saudi Arabia

${ }^{19}$ Adult Critical Care Department, King Faisal Specialist Hospital and Research Center, Riyadh, Saudi Arabia

${ }^{20}$ Pulmonary and Critical Care Departments, King Fahad Hospital Madinah, Madinah, Saudi Arabia

${ }^{21}$ Department of Critical Care Medicine, College of Medicine, King Saud University, Riyadh, Saudi Arabia

${ }^{22}$ Department of Intensive Care Services, Prince Sultan Military Medical City, Riyadh, Saudi Arabia

${ }^{23}$ Intensive Care Department, King Faisal Specialist Hospital and Research Centre, Jeddah, Saudi Arabia

${ }^{24}$ Department of Pulmonary and Critical Care Medicine, King Fahad Medical City, Riyadh, Saudi Arabia

${ }^{25}$ Department of Anesthesia and Critical Care, King Abdulaziz University Hospital, Jeddah, Saudi Arabia

${ }^{26}$ Department of Anesthesia, Critical Care Medicine and Pain Medicine, Al-Amiri Hospital, Kuwait

Twitter Mohammad Khulaif Al Harbi @MohammedAlras12

Acknowledgements The authors thank all the participating patients and their families, as well as the members of the Data Safety \& Monitoring Board: Chair: Dr Nicholas S. Hill (Professor of Medicine, Chief of Pulmonary, Critical Care and Sleep Division, Tufts Medical Centre, Boston, Massachusetts, USA); DSM members: Dr Stefano Nava (Professor of Respiratory Medicine University of Bologna, Chief of the Respiratory and Critical Care Sant' Orsola Hospital Bologna, Specialist in Respiratory Medicine and Intensive Care Medicine, University of Bologna, Italy); Dr James Mojica (Vice Chief and Clinical Director of Pulmonary \& Critical Care, Director, The Sleep Centre at Spaulding, Massachusetts General Hospital, USA) and Dr Michael Harhay (Assistant Professor of Epidemiology and Medicine-Pulmonary and Critical Care, Department of Biostatistics, Epidemiology and Informatics, University of Pennsylvania USA).

Contributors YMA is the principal investigator and participated in the project concept, design, final approval and manuscript preparation, review and submission. HT, SA, HMA-D, SAA, MKAH, HAH, MAM, OAZ, EAQ, WAW, SAQ, FA-H, JC, MAls, AAlh, AM, ABE, AAAb, ZAA, ZAD, AK, RA, AAG, AAlt, KA, MAIm, HA, AAA, FAB, HAS, MAO, YTI and AAA-F participated in the critical revision, final approval of the protocol and manuscript preparation, review and submission. All authors agree to be accountable for the accuracy and integrity of the work.

Funding The study is funded by King Abdullah International Medical Research Center (RC 20/306/R). The study sponsor does not have any role in the study design, collection, management, analysis and interpretation of data, as well as writing of the report.

Competing interests None declared.

Patient consent for publication Not required.

Provenance and peer review Not commissioned; externally peer reviewed.

Supplemental material This content has been supplied by the author(s). It has not been vetted by BMJ Publishing Group Limited (BMJ) and may not have been peer-reviewed. Any opinions or recommendations discussed are solely those of the author(s) and are not endorsed by BMJ. BMJ disclaims all liability and responsibility arising from any reliance placed on the content. Where the content includes any translated material, BMJ does not warrant the accuracy and reliability of the translations (including but not limited to local regulations, clinical guidelines, terminology, drug names and drug dosages), and is not responsible for any error and/or omissions arising from translation and adaptation or otherwise.

Open access This is an open access article distributed in accordance with the Creative Commons Attribution Non Commercial (CC BY-NC 4.0) license, which permits others to distribute, remix, adapt, build upon this work non-commercially, and license their derivative works on different terms, provided the original work is properly cited, appropriate credit is given, any changes made indicated, and the use is non-commercial. See: http://creativecommons.org/licenses/by-nc/4.0/. 


\section{ORCID iDs}

Yaseen M Arabi http://orcid.org/0000-0001-5735-6241

Ali A Al bshabshe http://orcid.org/0000-0003-0974-7226

\section{REFERENCES}

1 World Health Organization. Available: https://covid19.who.int/ [Accessed 17 Aug 2021].

2 Marini JJ, Gattinoni L. Management of COVID-19 respiratory distress. JAMA 2020;323:2329.

3 Guan W-J, Ni Z-Y, Hu Y, W-j G, Z-y N, et al. Clinical characteristics of coronavirus disease 2019 in China. N Engl J Med 2020;382:1708-20.

4 Ñamendys-Silva SA. Respiratory support for patients with COVID-19 infection. Lancet Respir Med 2020;8:e18.

5 Richardson S, Hirsch JS, Narasimhan M, et al. Presenting characteristics, comorbidities, and outcomes among 5700 patients hospitalized with COVID-19 in the new York City area. JAMA 2020;323:2052.

6 Xu X-P, Zhang X-C, Hu S-L. Noninvasive ventilation in acute hypoxemic Nonhypercapnic respiratory failure. Crit Care Med 2017;45.

7 Brambilla AM, Aliberti S, Prina E, et al. Helmet CPAP vs. oxygen therapy in severe hypoxemic respiratory failure due to pneumonia. Intensive Care Med 2014;40:942-9.

8 Arulkumaran N, Brealey D, Howell D, et al. Use of non-invasive ventilation for patients with COVID-19: a cause for concern? Lancet Respir Med 2020;8:e45.

9 Esquinas Rodriguez AM, Papadakos PJ, Carron M, et al. Clinical review: helmet and non-invasive mechanical ventilation in critically ill patients. Crit Care 2013;17:cc11875

10 Patroniti N, Foti G, Manfio A, et al. Head helmet versus face mask for non-invasive continuous positive airway pressure: a physiological study. Intensive Care Med 2003;29:1680-7.

11 Cosentini R, Brambilla AM, Aliberti S, et al. Helmet continuous positive airway pressure vs oxygen therapy to improve oxygenation in community-acquired pneumonia: a randomized, controlled trial. Chest 2010;138:114-20.

12 Patel BK, Wolfe KS, Pohlman AS, et al. Effect of noninvasive ventilation delivered by helmet vs face mask on the rate of endotracheal intubation in patients with acute respiratory distress syndrome: a randomized clinical trial. JAMA 2016;315:2435.

13 Foti G, Sangalli F, Berra L, et al. Is helmet CPAP first line pre-hospital treatment of presumed severe acute pulmonary edema? Intensive Care Med 2009;35:656-62.

14 Squadrone V, Coha M, Cerutti E, et al. Continuous positive airway pressure for treatment of postoperative hypoxemia: a randomized controlled trial. JAMA 2005;293:589.

15 Patel BK, Wolfe KS, MacKenzie EL, et al. One-Year outcomes in patients with acute respiratory distress syndrome enrolled in a randomized clinical trial of helmet versus facemask noninvasive ventilation. Crit Care Med 2018;46:1078-84.
16 Liu Q, Gao Y, Chen R, et al. Noninvasive ventilation with helmet versus control strategy in patients with acute respiratory failure: a systematic review and meta-analysis of controlled studies. Crit Care 2016;20:265.

17 Luo Y, Luo Y, Li Y. Helmet CPAP versus oxygen therapy in hypoxemic acute respiratory failure: a meta-analysis of randomized controlled trials, 2016

18 Ferreyro BL, Angriman F, Munshi L, et al. Association of noninvasive oxygenation strategies with all-cause mortality in adults with acute hypoxemic respiratory failure: a systematic review and meta-analysis. JAMA 2020;324:57-67.

19 Kyeremanteng K, Gagnon L-P, Robidoux R, et al. Cost analysis of noninvasive helmet ventilation compared with use of noninvasive face mask in ARDS. Can Respir J 2018;2018:6518572:1-5.

20 Grieco DL, Menga LS, Cesarano M, et al. Effect of helmet noninvasive ventilation vs high-flow nasal oxygen on days free of respiratory support in patients with COVID-19 and moderate to severe hypoxemic respiratory failure: the HENIVOT randomized clinical trial. JAMA 2021;325:1731.

21 Bellani G, Laffey JG, Pham T, et al. Epidemiology, patterns of care, and mortality for patients with acute respiratory distress syndrome in intensive care units in 50 countries. JAMA 2016;315:788.

22 Hasan SS, Capstick T, Ahmed R, et al. Mortality in COVID-19 patients with acute respiratory distress syndrome and corticosteroids use: a systematic review and meta-analysis. Expert Rev Respir Med 2020;14:1149-63.

23 Centers for Disease Control and Prevention. Coronavirus disease 2019 (COVID-19). 2020 interim case definition, Approved April 5, 2020. Available: https://wwwn.cdc.gov/nndss/conditions/ coronavirus-disease-2019-covid-19/case-definition/2020/ [Accessed 02 Jun 2020].

24 Arabi YM, Al Aseri Z, Alaama T, et al. National approach to standardize and improve mechanical ventilation. Ann Thorac Med 2019;14:101.

25 Alhazzani W, Al-Suwaidan F, Al Aseri Z, et al. The Saudi critical care Society clinical practice guidelines on the management of COVID-19 patients in the intensive care unit. Saudi Crit Care J 2020;4:27-44.

26 Alhazzani W, Evans L, Alshamsi F, et al. Surviving sepsis campaign guidelines on the management of adults with coronavirus disease 2019 (COVID-19) in the ICU: first update. Crit Care Med 2021;49:e219-34.

27 Alhazzani W, Møller MH, Arabi YM, et al. Surviving sepsis campaign: guidelines on the management of critically ill adults with coronavirus disease 2019 (COVID-19). Crit Care Med 2020;48:e440-69.

28 Coronavirus disease (COVID-19) technical guidance: patient management. Available: https://www.who.int/emergencies/diseases/ novel-coronavirus-2019/technical-guidance/patient-management [Accessed 1 Mar 2021].

29 Knaus WA, Draper EA, Wagner DP, et al. Apache II: a severity of disease classification system. Crit Care Med 1985;13:818-29.

30 Cook D, Lauzier F, Rocha MG, et al. Serious adverse events in academic critical care research. CMAJ 2008;178:1181-4. 